\section{MUCOCELE DO APÊNDICE}

\author{
MUCOCELE OF THE APPENDIX
}

\section{INTRODUÇÃO}

A mucocele do apêndice foi reconhecida como entidade patológica por Rokitansky em 1842 e formalmente denominada por Feren em $1876^{1}$.

Mucocele é um termo inespecífico para descrever uma rara lesão do apêndice que se apresenta distendido por acúmulo de muco dentro de sua luz, podendo ser procedente de tumor benigno como hiperplasia mucosa ou cistoadenoma mucinoso ou maligno como cistoadenocarcinoma mucinoso. O diagnóstico normalmente é feito no intra-operatório e raras vezes em achados ultra-sonográficos ou radiológicos ${ }^{2}$.

O objetivo desta publicação é apresentar um caso de mucocele de apêndice cujo diagnóstico foi feito no préoperatório.

\section{RELATO DO CASO}

Paciente do sexo feminino, branca, 73 anos, procurou ginecologista para exame preventivo quando lhe foi solicitada ultra-sonografia pélvica como rotina, que mostrava tumor reniforme em fossa ilíaca direita. A paciente procurou nosso serviço e apresentava-se completamente assintomática, corada, hidratada, normotensa, com passado de cesariana há 41 anos, herniorrafia inguinal direita recidivada há 20 anos e toracotomia para correção de aneurisma de aorta torácica e troca de válvula cardíaca há dois anos. Em uso de warfarina sódica $5 \mathrm{mg}$ por dia. Ao exame físico do abdome palpava-se tumor móvel, duro, indolor, de mais ou menos $15 \mathrm{~cm}$ de comprimento por $10 \mathrm{~cm}$ de diâmetro, em quadrante inferior direito. A tomografia computadorizada (TC) do abdome mostrou

\author{
José Carlos de Rezende Pereira, ACBC-RJ ${ }^{1}$ \\ Carlos Antônio da Silva Júnior, ACBC-RJ ${ }^{2}$
}

tumor de consistência líquida em seu interior, em contigüidade com a borda distal do ceco, e diagnóstico sugestivo de mucocele de apêndice.

No acompanhamento clínico pré-operatório foi suspensa a warfarina, sete dias antes da colonoscopia e 15 dias da cirurgia, sendo iniciada heparina de baixo peso molecular - enoxaparina em dose prófilática de $40 \mathrm{mg}$ por dia. Internada no mesmo dia da cirurgia, teve o preparo de cólon feito com manitol $500 \mathrm{ml}$, iniciado seis horas antes do procedimento cirúrgico. A profilaxia antibiótica foi feita com metronidazol 500mg e gentamicina $80 \mathrm{mg}$ - uma dose antes e três doses depois do ato operatório. Suspendemos a enoxiparina 12 horas antes e recomeçamos seis horas depois da cirurgia.

A paciente foi submetida à laparotomia com incisão mediana infra-umbilical. Encontramos apêndice vermiforme sem aderências, com $15 \mathrm{~cm}$ de comprimento, $10 \mathrm{~cm}$ no seu maior diâmetro e base livre com aproximadamente $8 \mathrm{~cm}$ de diâmetro. Após proteção da parede abdominal, optamos por fazer a apendicectomia, com tratamento da base do apêndice através da ressecção de $20 \%$ da face distal do ceco com grampeador TL 60 . A investigação da cavidade abdominal mostrou ovários normais e peritônio livre de implantes de mucina.

No pós-operatório a paciente evoluiu bem, iniciando dieta na 14a hora pós-cirurgia e alta hospitalar com 48 horas da internação, quando foi reiniciado anticoagulante oral.

O exame anatomopatológico corroborou com o laudo da biópsia colonoscópica de benignidade. Nos pacientes com concomitância de outras doenças com adenocarcinoma do cólon acima de 10\%, recomenda-se o acompanhamento com protocolo para adenocarcinoma colônico. Neste caso onde a concomitância atinge $20 \%$, achamos conveniente acompanhar com o protocolo supracitado.

1. Chefe do Serviço de Cirurgia do Programa Médico de Família de Niterói. Cirurgião do Hospital Santa Cruz de Niterói.

2. Cirurgião do Programa Médico de Família de Niterói. Cirurgião do Hospital Santa Cruz de Niterói.

Recebido em 9/3/2000

Aceito para publicação em 26/9/2000

Trabalho realizado no Hospital Santa Cruz de Niterói - RJ. 

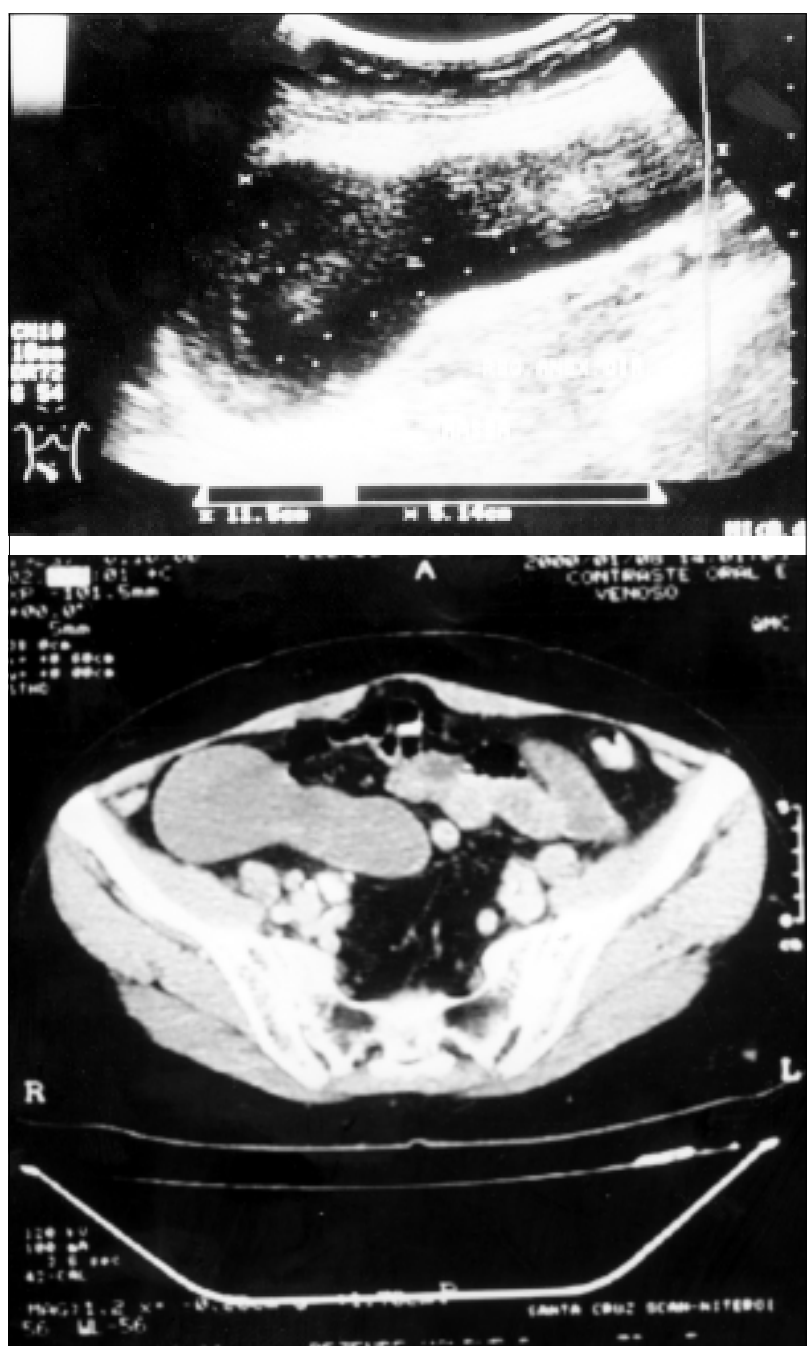

Figura 1 - US e tomografia mostrando tumor de consistência cística em fossa ilíaca direita.

\section{DISCUSSÃO}

A mucocele do apêndice aparece em $0,1 \%$ a $0,4 \%^{3}$ de todas as apendicectomias com predominância em mulheres com mais de 50 anos e sendo raro seu diagnóstico pré-operatório. Os sinais e sintomas, quando aparecem, são: dor em fossa ilíaca direita, alteração de trânsito intestinal, anemia, hematoquesia e, dependendo da localização do apêndice, sinais outros como hematúria ${ }^{4}$. A complicação mais temível é o pseudomixoma peritoneal, de difícil tratamento e prognóstico reservado, ocorrendo independente de a mucocele ser procedente de doença benigna ou maligna e com taxa de sobrevida de cinco anos de $53 \%$ a $75 \%$, variando de acordo com a doença primária ser benigna ou maligna ${ }^{5}$.

Como a manipulação exagerada ou inadequada durante $\mathrm{o}$ ato operatório pode deixar extravasar mucina na cavidade peritoneal livre, o tratamento por videolaparoscopia é contra-indicado, pois pode levar a implantes de tumor mucinoso difusamente sobre a superfície peri-
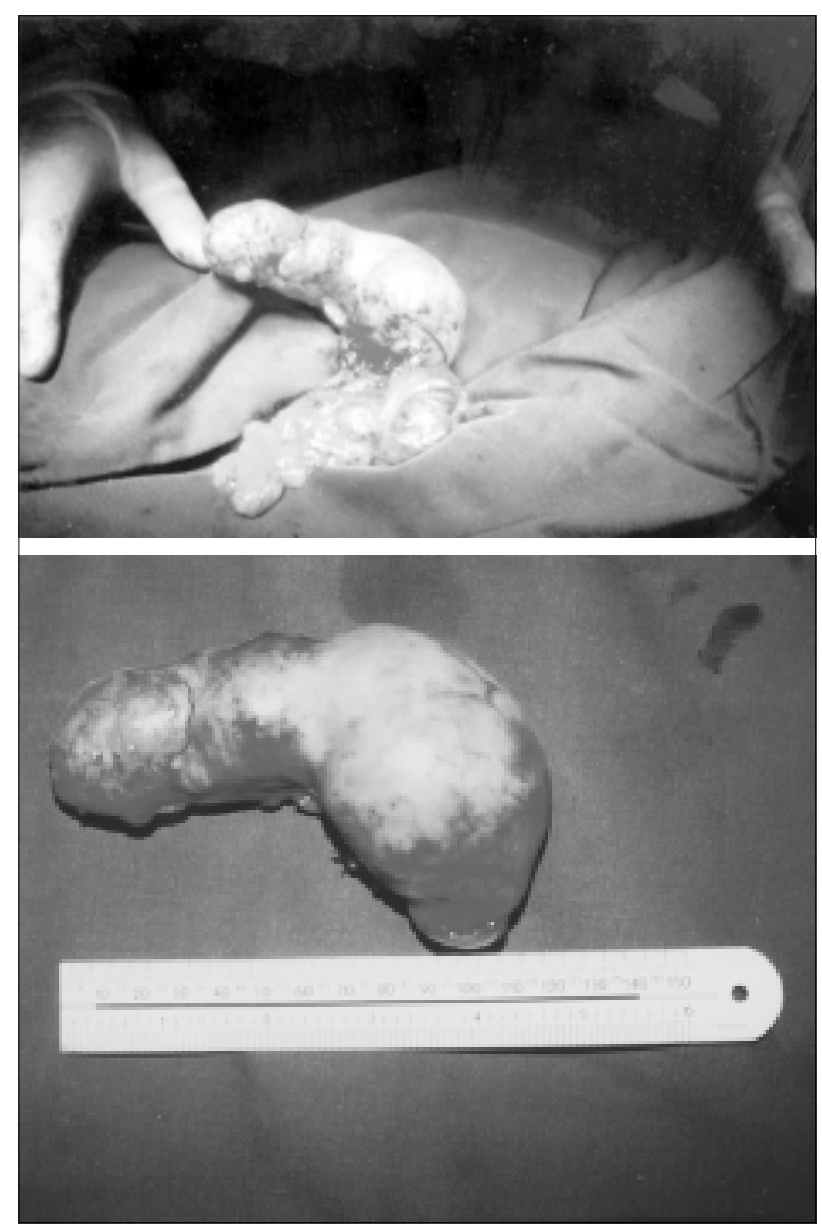

Figura 2 - Mucocele do apêndice.

toneal. Os autores sugerem: se em uma apendicectomia laparoscópica encontrarmos um tumor mucinoso de apêndice, devemos converter o procedimento para laparotomia e a apendicectomia ser totalmente atraumática.

A cirurgia normalmente indicada para mucocele do apêndice é a colectomia direita, mas a apendicectomia é técnica aceitável no caso de tumores benignos. No caso em questão, como o estudo pré-operatório mostrava benignidade e a base do apêndice livre de doença, consideramos a apendictomia, feita com grampeador TL 60, a menos traumática e a mais adequada para a paciente.

Este caso nos mostra que apesar de freqüentemente o diagnóstico de mucocele do apêndice ser acidental, um exame físico bem feito, a ultra-sonografia abdominal, a colonoscopia e principalmente a TC, podem sugerir o diagnóstico e ajudar na escolha da tática cirúrgica. A associação de adenocarcinoma do colo em $20 \%$ dos casos de mucocele de apêndice, e a correlação concomitante, em $13 \%$ dos casos, com cistoadenoma ou cistoadenocarcinoma de ovário, fazem do CEA e CA50, para pesquisa de carcinoma de colon in situ, assim como estudo ultra-sonográfico dos ovários, arsenal propedêutico armado para o prognóstico, e importantes no protocolo de acompanhamento pós-operatório. 


\begin{abstract}
A case of benign appendiceal mucocele treated by appendectomy tony is reported. Appendiceal mucocele is a rare lesion of the appendix, characterized by a gross enlargement of the appendix from luminal accumulation of mucoid substance. It is a rare condition, detected in only 0.1-0.4\% of all appendicectomies, with a female predominance and an average age at the time of diagnosis over 50 years. The possibility of a pre-operative diagnosis is examined. Abdominal ultrasound and CT scan of the abdomen or colonoscopy may suggest the diagnosis. However the diagnosis is often incidental. The pathogenesis and the different surgical strategies are discussed.
\end{abstract}

Key Words: Appendiceal mucoceles; Pre-operative diagnosis.

\section{REFERÊNCIAS}

1. Andriani AC, Araujo PA, Kestering DM, et al: Mucocele de apêndice e pseudomixoma peritoneal. Rev Col Bras Cir 1997 24(1): 441-2.

2. Kim SH, Lim HK, Lee WJ: Mucocele of the appendix: ultrasonographic and CT findings. Abdom Imaging 1998; 23(3): 292-6.

3. Emmi S, Gallasso MG, Ursino V, et al: Appendicial mucoceles. A case report. Minerva Chir 1998;53(10):80710.

4. Oliphant UJ, Rosenthal A: Hematuria: an unusual presentation for mucocele of the appendix. Case report and review of the literature. JSLS 1999; 3(1): 71-4.
5. Hinson FL, Ambrose NS: Pseudomyxoma peritonei. Br J Surg 1998; 85(10):1332-9.

Endereço para correspondência:

Dr. José Carlos de Rezende Pereira

Rua Madrid, 338

24355-020 - Niterói - RJ 